\title{
Final Scientific/Technical Report for DE-FG03-02NA00063 Coherent imaging of laser-plasma interactions using XUV high harmonic radiation
}

\author{
Prof. Henry C. Kapteyn \\ JILA, University of Colorado at Boulder, Boulder, CO 80309-0440 \\ Phone: (303) 492-8198; FAX: (303) 492-5235; E-mail: kapteyn@jila.colorado.edu
}

\section{Executive Summary}

The objective of this project was to develop experimental techniques for using coherent extremeultraviolet (EUV) radiation generated using the high-order harmonic generation technique, as an illumination source for studies of high-density plasmas relevant to the stockpile stewardship mission. In this project, we made considerable progress, including the first demonstration of imaging of dynamic processes using this coherent ultrashort pulse light. This work also stimulated considerable progress in the development of the required ultrashort EUV pulses, and in the development of new laser technologies that have been commercialized. We also demonstrated the first EUV sources that exhibit full intrinsic optical coherence. This work resulted in 12 publications.

\section{Highlights of Progress:}

1. Our group published 12 papers, received 3 major national awards, and obtained one patent in work partially or primarily supported by this grant.

2. Two people (one M.S. student and one postdoc) from our group took positions at DP Laboratories - Dr. Erez Gershgoren at LANL and Jason Schmidt at LLNL.

3. The high-power femtosecond laser system designs developed in our group during the past two grant periods under partial DOE/NNSA support are now in use in several DoE BES and NNSA/DP Research Labs.

4. Our work has made substantial progress toward the project objective of using coherent extreme-ultraviolet light to image high-density plasmas. We made substantial progress in understanding the plasma physics aspects of the physics of the high-order harmonic generation process used to generate coherent extreme-ultraviolet light. And we made the first dynamic images of plasma dynamics using a novel time-resolved imaging setup. Continuing work will make use of this setup to study the physics of high-density plasmas.

A detailed discussion of major results from this work is detailed below:

\section{Demonstration of fully spatially coherent EUV beams at $13 \mathrm{~nm}$ in plasmas}

In recent work, we demonstrated full spatial coherence of extreme ultraviolet (EUV) beams generated by the process of high harmonic upconversion of a femtosecond laser, at wavelengths around $11 \mathrm{~nm}$ and $13 \mathrm{~nm}$. It is important to note that this work to demonstrate full coherence around $13 \mathrm{~nm}$ was not merely an extension of our previous work where we demonstrated full spatial coherence at wavelengths around $30 \mathrm{~nm}$. The new work required a much more intensive experimental and theoretical study of the interaction of intense ultrashort laser beams with rapidly ionizing plasmas, and several unexpected results were obtained.

To obtain such a high-flux, fully coherent beam at $13 \mathrm{~nm}$, we first needed to improve phase matching techniques in ionized plasmas so that we could generate this wavelength more 


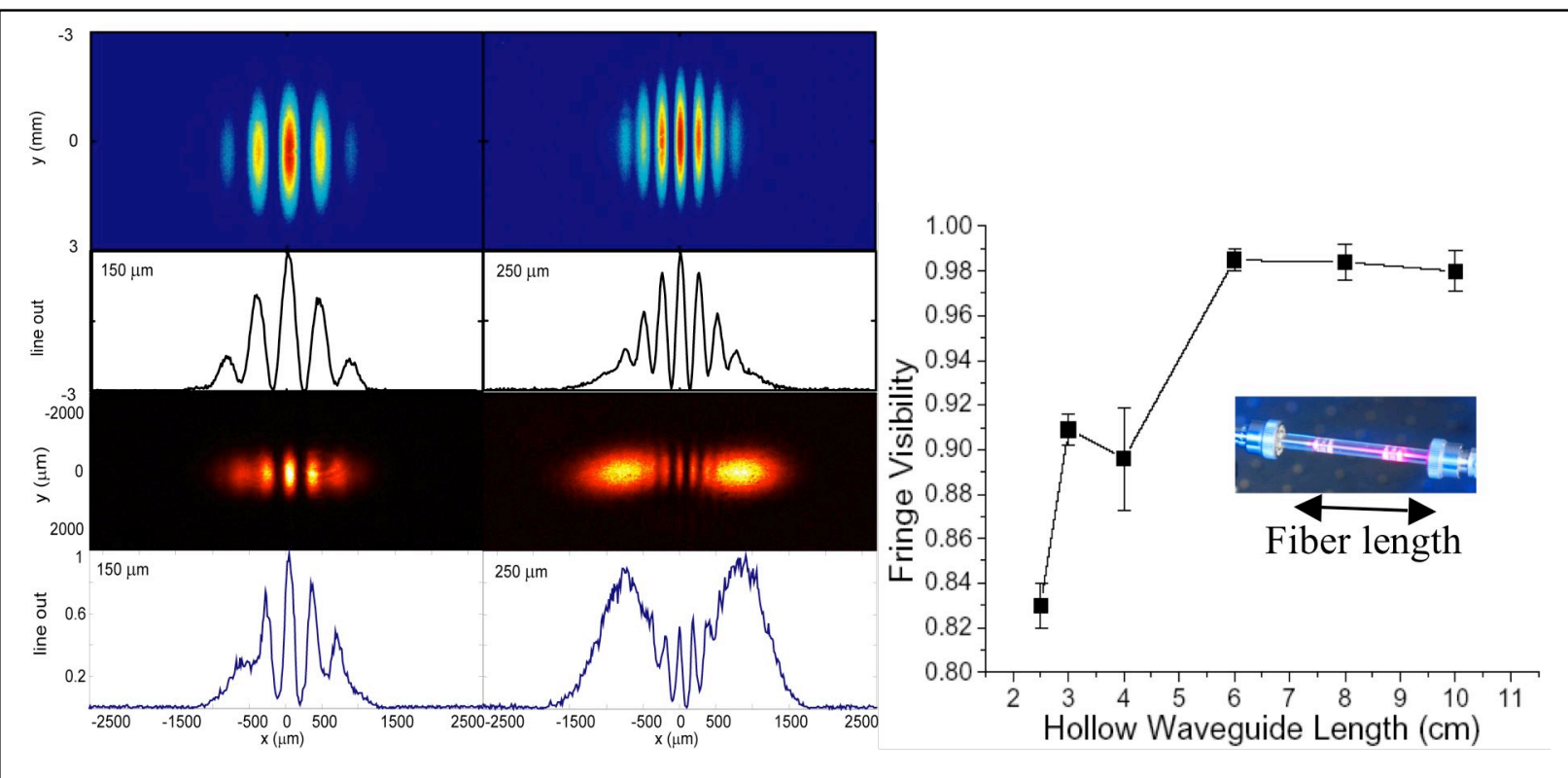

Figure 1. Top left: Interferograms and lineouts of a $1 \mathrm{~mm}$ EUV beam at $31 \mathrm{eV}$ center photon energy, A $10 \mathrm{~cm}$ long straight waveguide filled with 29 torr of Ar was used for this measurement. Bottom left: Interferograms and lineouts of a $1 \mathrm{~mm}$ EUV beam centered at $90 \mathrm{eV}$ center photon energy. A $10 \mathrm{~cm}$ long modulated waveguide, with periodicity of $0.5 \mathrm{~mm}$, and filled with 150 torr of $\mathrm{He}$ was used for this measurement. Pinhole pairs at separations of $150 \mu \mathrm{m}$ and $250 \mu \mathrm{m}$ were used for these measurements. The fringe contrast is $100 \%$ only at the center, because combs of 5-7 harmonics were used, and each harmonic has a slightly different interference pattern on the CCD camera. Right: Fringe visibility (spatial coherence) as a function of waveguide length for EUV beams generated in 40 torr of Argon plasma. Full spatial coherence is observed only when long interaction lengths $(>6 \mathrm{~cm})$ are observed. These are the highest intrinsic spatial coherence measurements of any EUV light source at such short wavelengths.

efficiently - even just in order to characterize the radiation. Experimentally, in work published in Nature and Science, we demonstrated quasi phase matching of high harmonic radiation using modulated waveguides to contain the gas medium, to extend the observable, bright, harmonic orders to shorter wavelengths.

The next step was to measure the spatial coherence of the EUV beams from these modulated waveguides, particularly at very short wavelengths around $11-13 \mathrm{~nm}$ that would enable higher spatial resolution using more efficient EUV optics. At high laser intensities and ionization levels, the laser beam is refracted by a plasma and continually re-guided by the modulations on the hollow modulated waveguide. Therefore, it was not at all certain that the output EUV beams would exhibit full spatial coherence compared with that obtained at wavelengths around 30nm where the gas is not highly ionized. The degree of spatial coherence can be determined by measuring the fringe visibility in a Young's double pinhole experiment as a function of pinhole separation across the EUV beam. Using this approach, in work published in Optics Letters and Applied Physics Letters, we demonstrated that phase matched and quasi-phase matched highharmonic generation in modulated waveguides does indeed produce EUV beams with full spatial coherence at wavelengths from $30 \mathrm{~nm}-13 \mathrm{~nm}$, provided that relatively long waveguides with lengths $>6 \mathrm{~cm}$ are used.

Figure 1 shows sample interferograms and their lineouts for $1 \mathrm{~mm}$ EUV beams centered at 30nm and $13 \mathrm{~nm}$, diffracted by pinhole pairs at separations of $150 \mu \mathrm{m}$ and $250 \mu \mathrm{m}$. Because broad bandwidth combs of 5-7 harmonics were used for this measurement, the fringe contrast is $100 \%$ only at the center, since each harmonic gives rise to a slightly different interference pattern on the CCD camera. This series of experiments demonstrated the highest spatial coherence values 
ever measured at such short wavelengths from any source without spatial filtering. Therefore, HHG-based EUV sources will be useful for applications in metrology, coherent imaging, nonlinear spectroscopies, and microscopy.

Figure 1 (right) also shows the measured fringe visibility (spatial coherence) as a function of waveguide length for EUV beams generated in 40 torr of Argon. It is clear that in order to generate fully spatially coherent extreme-ultraviolet (EUV) beams using HHG, the driving laser beam must be guided over relatively long interaction lengths $(\sim 10 \mathrm{~cm})$. To understand the evolution of the laser pulse and the EUV beam in the plasma contained within a modulated waveguide, our theory collaborator, Dr. Ivan Christov, used a fully space-time model to predict the propagation of an intense laser pulse through a multiply-ionizing plasma in a guided geometry. This model takes into account contributions to the laser and EUV pulse propagation due to diffraction, dispersion of the neutral atoms, dispersion due to the time-dependent plasma density, and loss due to ionization. Also, by applying appropriate boundary conditions for the hollow waveguide, the model can include the additional negative dispersion that is present in the guided geometry. When comparing the waveguide with a free-space geometry (i.e. HHG in a jet), the Guoy phase shift is automatically included in the calculations. Using this model we were thus able to follow the processes of phase matching in the hollow waveguide, and its dependence on the key parameters of the experiment. In particular, we can compare the spatial properties of the laser and EUV beams both in the free-focused and guided-wave geometries, shown in Fig. 2. Our radiation and plasma models were able to explain why full spatial coherence was observed only for long interaction lengths in a guided geometry.

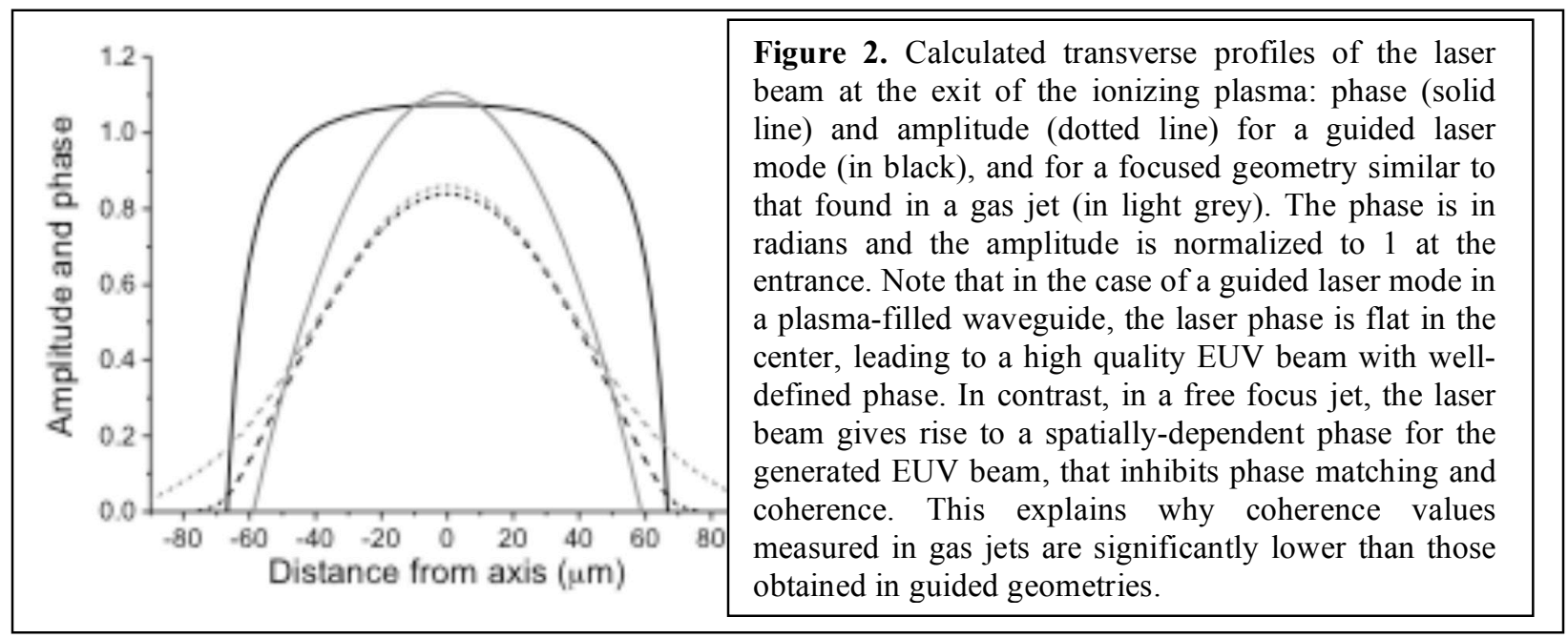

This work has had four important outcomes to date: 1) It uncovered a new regime of guiding a laser beam in a plasma-filled waveguide, where a stable eigenmode can be formed even in a plasma-filled waveguide. 2) As a result of being able to support a stable laser eignenmode in the plasma-filled waveguide, the generated EUV beams were perfectly spatially coherent. 3) In related work, also because of the support of a stable laser eigenmode in a plasma-filled waveguide, very high-order harmonic generation from ions was observed. 4) Finally, in other related work, a new pulse compression mechanism was identified, where the laser pulse emerges from the waveguide with a shorter pulse duration than when it entered without any need for dispersion compensation. The shorter pulse duration was due to rapid phase modulation in a rapidly ionizing plasma, which rapidly changes the index of refraction. When this effect is combined with plasma refraction and guiding in a waveguide, the leading edge of the laser pulse steepens, and the pulse duration is reduced by a factor of 2-3, from $30 \mathrm{fs}$ to $13 \mathrm{fs}$. This combined work also has exciting new possibilities for uncovering new ultrafast plasma physics, as well as having consequences for coherent EUV source technologies. 


\section{High-resolution static and dynamic imaging using an EUV microscope}

The ultimate goal of the project was to apply EUV sources to high-resolution imaging of highdensity, warm or turbulent plasmas. This required development of a long-working-distance EUV microscope based on multilayer optics. We first implemented high-resolution static imaging of small objects, using the set-up shown in Figure 3. A static grid with $10 \mu \mathrm{m}$ thick wires was inserted instead of the droplet source, and positioned about $50 \mathrm{~cm}$ from the EUV source. Two multilayer mirrors were used to produce a x50-magnified image on an X-ray CCD camera. The first mirror had a focal length of $50 \mathrm{~cm}$, and was positioned about $58 \mathrm{~cm}$ from the object, at a magnification of $x 7$. Figure 4 shows the magnified image of a 10-micron grid taken at a magnification of $x 50$. Changing the distance between the EUV mirrors and between the mirrors and the CCD camera varies the magnification. The smallest resolvable feature size in theory is $13.5 \mu \mathrm{m} / 50$ or $270 \mathrm{~nm}$. However, in these images, we were limited by the numerical aperture of the system, and therefore the actual resolution was $\sim 1$ micron. Further optimization of the setup should yield even higher resolution in the near future.

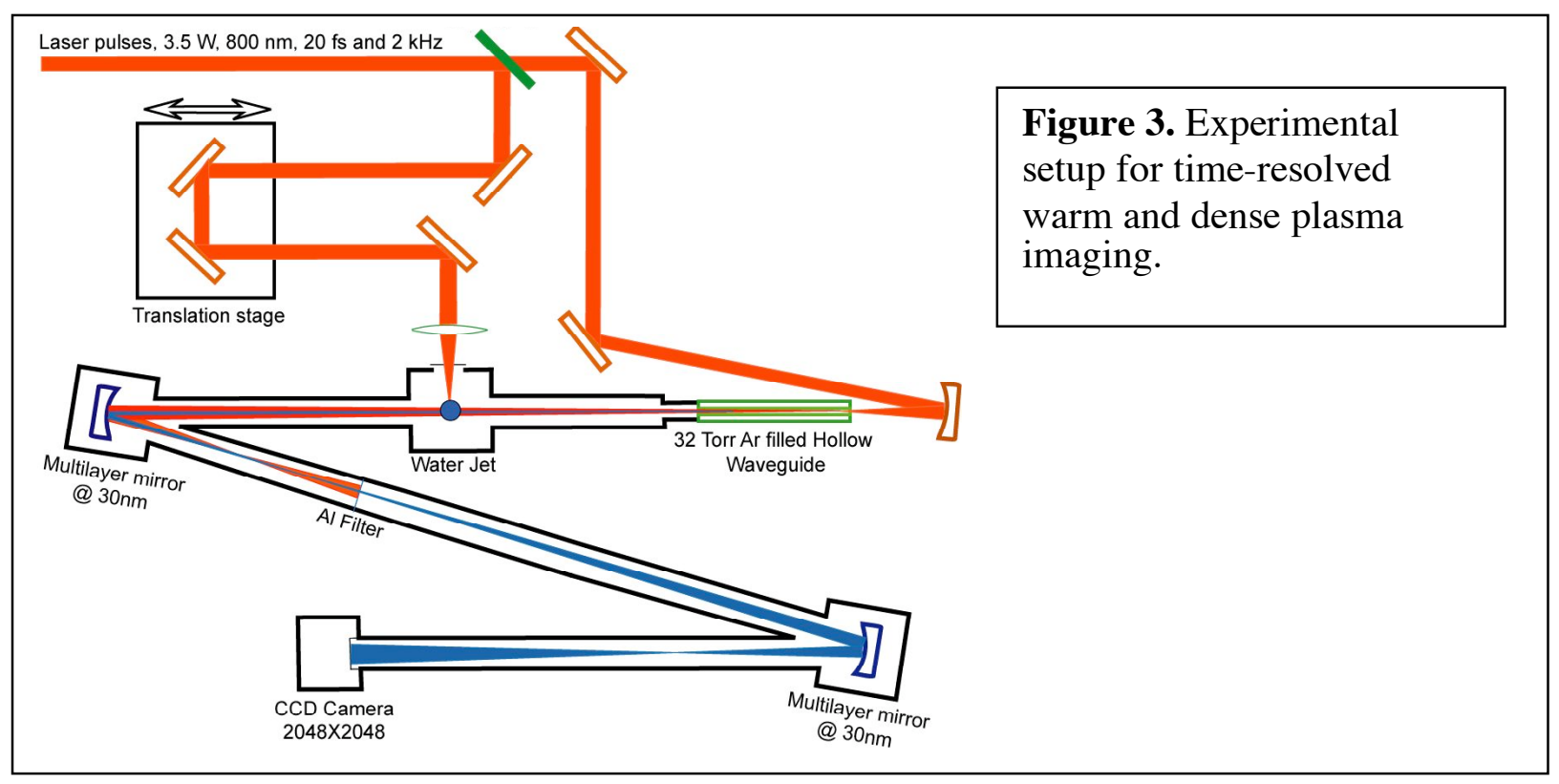

For dynamic imaging of plasmas, we developed a new synchronized droplet source that is now in operation. Droplet plasmas provide us with more opportunities for imaging turbulent and colliding plasmas than simply imaging a gas or liquid jet. We obtained the first sequence of images of ultrafast plasma dynamics taken using coherent EUV light at a wavelength of 30nm. Laser pulses at a repetition rate of $1-2 \mathrm{kHz}$ generated the EUV beams used for illumination, as well as for perturbing the water droplet to observe dynamics. In a series of images shown in the lower image of Fig. 4, three droplets can be seen (top to bottom) in each frame, with the center drop illuminated by $\sim 100$ microjoules of energy from the ultrafast laser. With varying pumpprobe delay, the expansion of the plasma can be observed. These are stroboscopic images, with each pump-probe delayed image taken over many thousands of laser shots. The jitter in droplet position is estimated from coherent artifacts in the data to be on order of 100nn.

This series of experiments has proven the basic feasibility of the proposed experiments and shown that we should be able to observe turbulent and colliding plasmas by illuminating two droplets at once. However, to image the critical density fronts and to gain more detailed information, we need to increase the spatial resolution of these images. The current series of experiments ran into a number of challenges, including understanding timing-stabilization of the droplet source, and (most significantly) developing alignment and focusing methodologies for a 
totally vacuum-enclosed imaging system spanning rather large ( $\sim 4$ meters end-end) distances between the mirrors (necessitated by the large working distance requirement in the case of plasma imaging).

\begin{tabular}{|l|l|l|}
\hline Eox & $\begin{array}{l}\text { Figure 4. Static and dynamic images } \\
\text { taken with an ult rafast EUV beam at } \\
\text { a wavelength of 30nm. (Top left) } \\
\text { Magnified image of a 10 micron grid } \\
\text { taken at a magnification of x50, in a } 3 \\
\text { minute exposure at a 2k Hz repetition } \\
\text { rate. In theses images, we are limited } \\
\text { by the numer ical aperture of the } \\
\text { system optics to a resolution just } \\
\text { below 1 micron. (Top right) Static } \\
\text { and dynamic images of a plasma } \\
\text { droplet, before and after irradiation } \\
\text { with a femtosecond laser. (Bottom) } \\
\text { Series of images of a plasma droplet } \\
\text { explosion induced by an ultrafast } \\
\text { laser. }\end{array}$ \\
\hline 0
\end{tabular}

\section{Products developed under the award and technology transfer activities}

\section{Publications as a Result of Award}

1. Henry C. Kapteyn, Margaret M. Murnane and Ivan P. Christov, "Coherent X-Rays from Lasers: Applied Attosecond Science", invited article, Physics Today, page 39 (March 2005).

2. E. A. Gibson, X. S. Zhang, T. Popmintchev, A. Paul, N. Wagner, A. Lytle, I. P. Christov, M. M. Murnane, and H. C. Kapteyn, "Extreme nonlinear optics: Attosecond photonics at short wavelengths," IEEE Journal Of Selected Topics In Quantum Electronics, vol. 10, pp. 13391350, 2004.

3. X. Zhang, A. R. Libertun, A. J. Paul, M. M. Murnane, H. Kapteyn, Y. Liu, and D. Attwood, "Coherent imaging of laser-plasma interactions using high-harmonic EUV light," in Ultrafast Phenomena XIV, Springer Series in Chemical Physics, T. Kobayashi, T. Okada, T. Kobayashi, K. A. Nelson, and S. D. Silvestri, Eds. Niigata, Japan: Springer-Verlag, 2004.

4. X. Zhang, A. R. Libertun, A. Paul, E. Gagnon, S. Backus, I. P. Christov, M. M. Murnane, H. C. Kapteyn, R. A. Bartels, Y. Liu, and D. T. Attwood, "Highly coherent light at $13 \mathrm{~nm}$ generated by use of quasi-phase-matched high-harmonic generation," Optics Letters 29, 1357 (2004).

5. Libertun, X. Zhang, A. Paul, E. Gagnon, T. Popmintchev, S. Backus, M. Murnane, H. Kapteyn, I. Christov, "Design of fully spatially coherent extreme-ultraviolet light sources," Appl. Phys. Lett. 84, 3903 (2004.) 
6. X. Zhang, D. Raymondson, A. S. Sandhu, S. Backus, M. M. Murnane, H. C. Kapteyn, and A. R. Libertun, "High-resolution imaging system using a tabletop extreme ultraviolet source," in Proceedings of the SPIE, vol. 5534. Bellingham, WA: SPIE, 2004, pp. 47-52.

7. Ariel Paul, Randy Bartels, Ivan Christov, Henry Kapteyn, Margaret Murnane, Sterling Backus, "Multiphoton photonics: quasi phase matching in the EUV", Nature 421, 51 (2003).

8. R. Bartels, A. Paul, S. Backus, H Kapteyn, M. Murnane, "EUV Photonics: Quasi Phase matching at Short Wavelengths", OSA Proc. Ultrafast Phenomena XIII (Springer Series in Chemical Physics), page 51 (2003).

9. R. Bartels, S, Backus, A. Paul, H Kapteyn, M. Murnane, "Determination of the HHG spectrum by measuring the mutual coherence", OSA Proc. Ultrafast Phenomena XIII (Springer Series), page 55 (2003).

10. R. Bartels, S. Backus, A. Paul, H Kapteyn, M. Murnane, Y. Liu, D. Attwood, C. Jacobsen "Fully spatially coherent EUV source", OSA Proc. Ultrafast Phenomena XIII (Springer Series), page 66 (2003).

11. R. Bartels, A. Paul, M. M. Murnane, H. C. Kapteyn, and S. Backus, "Absolute determination of the wavelength and spectrum of an EUV beam using a Young's double slit measurement", Optics Letters 27, 707 (2002).

12. R. Bartels, A. Paul, H. Green, H. Kapteyn, M. Murnane, S. Backus, I. Christov, Y. Liu, D. Attwood, C. Jacobsen, "Generation of spatially coherent light at extreme ultraviolet wavelengths," Science 297, 376 (2002).

\section{Patents}

- S. J. Backus, H. C. Kapteyn, and M. M. Murnane, "U.S. Patent \#6,804,287: Ultrashort pulse amplification in cryogenically cooled amplifiers," The Regents of the University of Colorado, 2004.

\section{Personnel supported by and collaborating on grant}

Professor Henry Kapteyn (no salary support)

Professor Margaret Murnane (no salary support)

Ariel Paul, graduate student (full salary support)

Ryan Anderson, graduate student (partial salary support)

Dr. Sterling Backus, Research Scientist (partial salary support)

Daisy Raymondson, graduate student (full salary support)

Dr. Oren Cohen (partial salary support since has partial fellowship for Yr 1 of postdoc)

James Holtsnider (partial salary support) 


\section{Interactions with National Laboratories and Government Agencies}

We have been and will continue to interact with DP personnel as part of this project. In particular, we summarize the following interactions and plans -

- We have coauthored a number of papers with DOE personnel. (D. Attwood, E. Gullikson from LBL)

- We attended three DOE and DOD workshops on homeland security, directed energy, and EUV light sources.

- Our group presented the Physics Colloquium at Los Alamos National Laboratories. We made contact with Dr. Jim Glownia during that visit, and have planned a series of complementary experiments on the same materials using different $\mathrm{x}$-ray and EUV probes. These experiments are discussed in this proposal. Because of the proximity of LANL to Boulder, we planned a series of visits to facilitate interactions.

- We invited Dr. Antoinette Taylor from Los Alamos National Laboratories to present the Physics Colloquium at Boulder, and to expose our large graduate program ( $<214$ students) to work being done at DP Labs. In particular, we have an Optical Science and Engineering $\mathrm{Ph} . \mathrm{D}$. program already in place at $\mathrm{CU}$ that requires students to do part of their Ph.D. work outside of $\mathrm{CU}$ to broaden their educational experience. This program provides one very convenient mechanism for student interaction with the DP labs. A group of students from physics/chemistry and engineering at CU toured labs at LANL in fall 2004.

- Our student from our group who is working on this project, Daisy Raymondson, will attend the HEDS (High Energy Density Science) Summer School at Berkeley in August. This workshop will take place in August 2005, and is sponsored by LLNL and Rochester. This student has already worked for one year at LLNL.

- One student from our group, Jason Schmidt, is now working at LLNL. A postdoc from our group, Dr. Erez Gershgoren, is now working at LANL.

- We had extensive interactions with scientists from DP Labs who were interested in using the high average power laser technology developed in our labs. Three laser systems will be in use at DP Labs in the near future.

\section{Impact on Technology and Personnel at DP Labs}

Using designated equipment funds of $\$ 90 \mathrm{~K}$ specially granted for this purpose, we upgraded and refurbished our existing amplified femtosecond laser to replace a very old pump laser as well as component parts. This upgrade was a great success - we were able to increase the laser output energy from $1 \mathrm{~mJ}$ to $3 \mathrm{~mJ}$, while decreasing the pulse duration from $28 \mathrm{fs}$ to $21 \mathrm{fs}$ (18fs best performance), while maintaining a repetition rate of $1 \mathrm{kHz}$. This technology had been transferred to industry though the Colorado Technology Transfer Office. Three laser systems based on our designs are now either already in use, or will soon be in use, at DP labs for a range of defenserelated applications. Therefore, the impact on DP manpower and technology from our grant was quite significant. 